Research Article

\title{
Structures and Low Dimensional Classifications of Hom-Poisson Superalgebras
}

\author{
Qingcheng Zhang, ${ }^{1}$ Chunyue Wang, ${ }^{2}$ and Zhu Wei ${ }^{1}$ \\ ${ }^{1}$ School of Mathematics and Statistics, Northeast Normal University, Changchun 130024, China \\ ${ }^{2}$ School of Applied Sciences, Jilin Engineering Normal University, Changchun 130052, China \\ Correspondence should be addressed to Chunyue Wang; wanglchun2yue3@163.com
}

Received 4 May 2015; Accepted 4 August 2015

Academic Editor: Hoshang Heydari

Copyright ( 2015 Qingcheng Zhang et al. This is an open access article distributed under the Creative Commons Attribution License, which permits unrestricted use, distribution, and reproduction in any medium, provided the original work is properly cited.

Hom-Poisson superalgebras can be considered as a deformation of Poisson superalgebras. We prove that Hom-Poisson superalgebras are closed under tensor products. Moreover, we show that Hom-Poisson superalgebras can be described using only the twisting map and one binary operation. Finally, all algebra endomorphisms on 2-dimensional complex Poisson superalgebras are computed, and their associated Hom-Poisson superalgebras are described explicitly.

\section{Introduction}

Poisson algebras are used in many fields in mathematics and physics. In mathematics, Poisson algebras play a fundamental role in Poisson geometry [1], quantum groups [2], and deformation of commutative associative algebras. In physics, Poisson algebras are a major part of deformation quantization, Hamiltonian mechanics [3], and topological field theories [4]. Poisson-like structures are also used in the study of vertex operator algebras [5]. Poisson superalgebras can be seen as a direct generalization of Poisson algebras. Remm show that a Poisson superalgebra can be described using only one binary operation in $[6,7]$.

Recently, a twisted generalization of noncommutative Poisson algebras, called Hom-noncommutative Poisson algebras, are studied in [8]. In a noncommutative Hom-Poisson algebra, there exists a twisted map, a Hom-Lie bracket and a Hom-associative product. The associativity, the Jacobi identity, and the Leibniz identity are considered as their Hom-type analogues in a noncommutative Hom-Poisson algebra. The purpose of this paper is to introduce and study a twisted generalization of Poisson superalgebras, called HomPoisson superalgebras.
This paper is organized as follows. In Section 2, we recall the construction of the Hom-Lie superalgebras. In Section 3, we give the definition of Hom-Poisson superalgebras. We show that starting with a Poisson superalgebra and an even Poisson superalgebra endomorphism, a Hom-Poisson superalgebra can be constructed. Moreover, we prove that HomPoisson superalgebras are closed under tensor products. In Section 4, we show that a Hom-Poisson superalgebra can be described using only the twisting map and one binary operation. Section 5 is devoted to classifying all the algebra endomorphisms $\alpha$ on all the 2-dimensional complex Poisson superalgebras and 2-dimensional Hom-Poisson superalgebras.

Throughout the paper $\mathbb{C}$ is the field of complex numbers. All algebras and vector spaces are considered over $\mathbb{C}$.

\section{Hom-Lie and \\ Hom-Associative Superalgebras}

In this section, we first recall the notion of Hom-Lie superalgebras and then give some construction of the Hom-Lie superalgebras. 
Definition 1 (see [9]). A Hom-associative superalgebra is a triple $(A, \circ, \alpha)$ consisting of a $\mathbb{Z}_{2}$-graded vector space $A$, an even bilinear map $\circ: A \times A \rightarrow A$, and an even homomorphism of algebras $\alpha: A \rightarrow A$ satisfying

$$
\alpha(x) \circ(y \circ z)=(x \circ y) \circ \alpha(z)
$$

for all homogeneous elements $x, y, z \in A$.

Definition 2 (see [9]). A Hom-Lie superalgebra is a triple $(A,[\cdot],, \alpha)$ consisting of a $\mathbb{Z}_{2}$-graded vector space $A$, an even bilinear map $[\cdot, \cdot]: A \times A \rightarrow A$ and an even homomorphism of algebras $\alpha: A \rightarrow A$ satisfying

$$
\begin{aligned}
& {[x, y]=-(-1)^{|x||y|}[y, x],} \\
& (-1)^{|x||z|}[\alpha(x),[y, z]]+(-1)^{|z||y|}[\alpha(z),[x, y]] \\
& \quad+(-1)^{|x||y|}[\alpha(y),[z, x]]=0
\end{aligned}
$$

for all homogeneous elements $x, y, z \in A$.

Let $(V,[\cdot, \cdot], \alpha)$ and $\left(V^{\prime},[\cdot, \cdot]^{\prime}, \alpha^{\prime}\right)$ be two Hom-Lie superalgebras. An even homomorphism $f: V \rightarrow V^{\prime}$ is said to be a morphism of Hom-Lie superalgebras if

$$
\begin{aligned}
{[f(x), f(y)]^{\prime} } & =f([x, y]), \quad \forall x, y \in V, \\
f \circ \alpha & =\alpha^{\prime} \circ f .
\end{aligned}
$$

Morphisms of Hom-associative superalgebras are defined similarly.

The following theorem provides a method to construct a Hom-Lie superalgebra by a Lie superalgebra and an even homomorphism of Lie superalgebras.

Proposition 3 (see [9]). Let $(V,[\cdot, \cdot])$ be a Lie superalgebra and let $\alpha: V \rightarrow V$ be an even endomorphism of Lie superalgebras. Then $\left(V,[\cdot, \cdot]_{\alpha}, \alpha\right)$ is a Hom-Lie superalgebra, where $[x, y]_{\alpha}=$ $\alpha([x, y])$.

Moreover, suppose that $\left(V^{\prime},[\cdot, \cdot]^{\prime}\right)$ is another Lie superalgebra and $\alpha^{\prime}: V^{\prime} \rightarrow V^{\prime}$ is an even endomorphism of Lie superalgebras. If $f: V \rightarrow V^{\prime}$ is a morphism of Lie superalgebras satisfying $f \circ \alpha=\alpha^{\prime} \circ f$, then

$$
f:\left(V,[\cdot, \cdot]_{\alpha}, \alpha\right) \longrightarrow\left(V^{\prime},[\cdot, \cdot]_{\alpha^{\prime}}^{\prime}, \alpha^{\prime}\right)
$$

Example 4 (see [9]). From the orthosymplectic Lie superalgebra $\operatorname{osp}(1,2)=V_{0} \oplus V_{1}$, where $V_{0}$ is spanned by

$$
\begin{aligned}
& H=\left(\begin{array}{lll}
1 & 0 & 0 \\
0 & 0 & 0 \\
0 & 0 & -1
\end{array}\right), \\
& X=\left(\begin{array}{lll}
0 & 0 & 1 \\
0 & 0 & 0 \\
0 & 0 & 0
\end{array}\right), \\
& Y=\left(\begin{array}{lll}
0 & 0 & 0 \\
0 & 0 & 0 \\
1 & 0 & 0
\end{array}\right),
\end{aligned}
$$

and $V_{1}$ is spanned by

$$
\begin{aligned}
& F=\left(\begin{array}{lll}
0 & 0 & 0 \\
1 & 0 & 0 \\
0 & 1 & 0
\end{array}\right), \\
& X=\left(\begin{array}{ccc}
0 & 1 & 0 \\
0 & 0 & -1 \\
0 & 0 & 0
\end{array}\right) .
\end{aligned}
$$

The defining nonzero relations are

$$
\begin{aligned}
& {[H, X]=2 X,} \\
& {[H, Y]=-2 Y,} \\
& {[X, Y]=H,} \\
& {[Y, G]=F,} \\
& {[X, F]=G,} \\
& {[H, F]=-F,} \\
& {[H, G]=G,} \\
& {[G, F]=H,} \\
& {[G, G]=-2 X,} \\
& {[F, F]=2 Y .}
\end{aligned}
$$

Let $\lambda \in \mathbb{R}^{*}$ define a linear map $\alpha_{\lambda}: \operatorname{osp}(1,2) \rightarrow \operatorname{osp}(1,2)$ by

$$
\begin{aligned}
& \alpha_{\lambda}(X)=\lambda^{2} X, \\
& \alpha_{\lambda}(Y)=\frac{1}{\lambda^{2}}(Y), \\
& \alpha_{\lambda}(H)=H, \\
& \alpha_{\lambda}(F)=\frac{1}{\lambda}(F), \\
& \alpha_{\lambda}(G)=\lambda G ;
\end{aligned}
$$


then $\alpha_{\lambda}$ is an even Lie superalgebra automorphism; by Proposition 3, we obtain a family of Hom-Lie superalgebras $\left(\operatorname{osp}(1,2),[,]_{\lambda}, \alpha_{\lambda}\right)$. These Hom-Lie superalgebras are not Lie superalgebras for $\lambda \neq 1$.

\section{Hom-Poisson Superalgebras}

Definition 5. A Hom-Poisson superalgebra is a tuple $(A, \cdot$, $[\cdot, \cdot], \alpha)$ consisting of a $\mathbb{Z}_{2}$-graded vector space $V$, two even bilinear maps $\cdot[\cdot[\cdot, \cdot]: V \times V \rightarrow V$, and an even homomorphism of algebras $\alpha: V \rightarrow V$, where $\alpha(x \cdot y)=\alpha(x) \cdot \alpha(y)$, $\alpha([x, y])=[\alpha(x), \alpha(y)]$, satisfying the following axioms.

(1) $(A, \cdot, \alpha)$ is a supercommutative Hom-associative superalgebra.

(2) $(A,[\cdot, \cdot], \alpha)$ is a Hom-Lie superalgebra.

(3) The Hom-Leibniz superidentity $[x \cdot y, \alpha(z)]=\alpha(x)$. $[y, z]+(-1)^{|y||z|}[x, z] \cdot \alpha(y)$ holds for all homogeneous elements $x, y, z \in A$.

Definition 6. Let $(A, \cdot,[\cdot, \cdot], \alpha)$ and $\left(A^{\prime}, \bullet^{\prime},[\cdot, \cdot]^{\prime}, \alpha^{\prime}\right)$ be two Hom-Poisson superalgebras. An even homomorphism $f$ : $A \rightarrow A^{\prime}$ is said to be a morphism of Hom-Poisson superalgebras if

$$
\begin{aligned}
f(x) \cdot f(y) & =f(x \cdot y), \quad \forall x, y \in A, \\
{[f(x), f(y)]^{\prime} } & =f([x, y)], \quad \forall x, y \in A, \\
f \cdot \alpha & =\alpha^{\prime} \cdot f .
\end{aligned}
$$

Proposition 7. Let $(A, \cdot, \alpha)$ be a Hom-associative superalgebra and $[\cdot, \cdot]: A \times A \rightarrow A$ be a binary operation on $A$ defined by

$$
[x, y]=x \cdot y-(-1)^{|x||y|} y \cdot x, \quad \forall x, y \in A,
$$

then $(A, \cdot,[\cdot, \cdot], \alpha)$ is a Hom-Poisson superalgebra with the same twisting map $\alpha$.

Proof. It is straightforward.

From Proposition 7, there is the following construction of Hom-Poisson superalgebras by Poisson superalgebras and homomorphisms.

Theorem 8. Let $(A, \cdot,[\cdot, \cdot])$ be a Poisson superalgebra and let $\alpha: A \rightarrow A$ be an even endomorphism of Poisson superalgebras. Then $\left(A,{ }_{\alpha},[\cdot, \cdot]_{\alpha}, \alpha\right)$ is a Hom-Poisson superalgebra, where $x_{\alpha} y=\alpha(x \cdot y)$ and $[x, y]_{\alpha}=\alpha([x, y])$.

Moreover, suppose that $\left(A^{\prime},{ }^{\prime},[\cdot, \cdot]^{\prime}\right)$ is another Poisson superalgebra and $\alpha^{\prime}: A^{\prime} \rightarrow A^{\prime}$ is an even endomorphism of Poisson superalgebras. If $f: A \rightarrow A^{\prime}$ is a morphism of Poisson superalgebras satisfying $f \cdot \alpha=\alpha^{\prime} \cdot f$, then

$$
f:\left(A,{ }_{\alpha},[\cdot, \cdot]_{\alpha}, \alpha\right) \longrightarrow\left(A^{\prime}, \cdot_{\alpha^{\prime}},[\cdot, \cdot]_{\alpha^{\prime}}^{\prime}, \alpha^{\prime}\right)
$$

is a morphism of Hom-Poisson superalgebras.

Proof. By Proposition $3,\left(A,[\cdot, \cdot]_{\alpha}, \alpha\right)$ is a Hom-Lie superalgebra.
We will show that $\left(A,{ }_{\alpha}, \alpha\right)$ satisfies the axioms (1) and (3) of Definition 5. In fact

$$
\begin{aligned}
\alpha(x) \cdot{ }_{\alpha}\left(y \cdot{ }_{\alpha} z\right)= & \alpha(x) \cdot{ }_{\alpha} \alpha(y \cdot z)=\alpha(\alpha(x) \cdot(y \cdot z)) \\
= & \alpha((x \cdot y) \cdot \alpha(z))=\alpha(x \cdot y) \cdot{ }_{\alpha} \alpha(z) \\
= & \left(x \cdot{ }_{\alpha} y\right) \cdot{ }_{\alpha} \alpha(z), \\
{\left[x_{\alpha} y, \alpha(z)\right]_{\alpha}=} & \alpha\left(\left[x \cdot{ }_{\alpha} y, \alpha(z)\right]\right) \\
= & \alpha([\alpha(x \cdot y), \alpha(z)]) \\
= & \alpha^{2}([x \cdot y, z]) \\
= & \alpha^{2}\left((-1)^{|y||z|}[x, z] \cdot y+x \cdot[y, z]\right) \\
= & (-1)^{|y||z|} \alpha(\alpha([x, z]) \cdot \alpha(y)) \\
& +\alpha(\alpha(x) \cdot \alpha([y, z])) \\
= & (-1)^{|y||z|} \alpha([x, z]) \cdot{ }_{\alpha} \alpha(y) \\
& +\alpha(x) \cdot{ }_{\alpha} \alpha([y, z]) \\
= & (-1)^{|y||z|}[x, z]_{\alpha} \cdot{ }_{\alpha} \alpha(y)+\alpha(x) \\
& { }_{\alpha}[y, z]_{\alpha} \cdot
\end{aligned}
$$

Hence $\left(A,{ }_{\alpha},[\cdot, \cdot]_{\alpha}, \alpha\right)$ is a Hom-Poisson superalgebra. Then second assertion follows from

$$
\begin{aligned}
f\left([x, y]_{\alpha}\right) & =f(\alpha([x, y]))=f \cdot \alpha([x, y]) \\
& =\alpha^{\prime} \cdot f([x, y])=\alpha^{\prime}([f(x), f(y)]) \\
& =[f(x), f(y)]_{\alpha^{\prime}} ; \\
f\left(x \cdot{ }_{\alpha} y\right) & =f(\alpha(x \cdot y))=f \cdot \alpha(x \cdot y) \\
& =\alpha^{\prime} \cdot f(x \cdot y)=\alpha^{\prime}(f(x) \cdot f(y)) \\
& =f(x) \cdot{ }_{\alpha^{\prime}} f(y) .
\end{aligned}
$$

This theorem provides a method to construct a HomPoisson superalgebra by a Poisson superalgebra and an even homomorphism of Poisson superalgebras.

Example 9. Let $A=A_{\overline{0}} \oplus A_{\overline{1}}$ be a 2-dimensional $\mathbb{Z}_{2}$-graded vector space, where $A_{\overline{0}}$ is generated by $e_{1}$ and $A_{\overline{1}}$ is generated by $e_{2}$ and nonzero products are given by

$$
\begin{aligned}
& e_{1} \cdot e_{1}=e_{1}, \\
& e_{2} \cdot e_{2}=e_{1}, \\
& e_{1} \cdot e_{2}=e_{2} \cdot e_{1}=e_{2}, \\
& {\left[e_{2}, e_{2}\right]=2 e_{1} ;}
\end{aligned}
$$

then $(A, \cdot,[\cdot, \cdot])$ is a Poisson superalgebra. For any $a \in \mathbb{C}$, we consider the homomorphism $\alpha: A \rightarrow A$ defined by $\alpha\left(e_{1}\right)=a e_{1}, \alpha\left(e_{2}\right)=a e_{2}$. By Theorem 8 , for any $a \in \mathbb{C}$, 
there is the corresponding Hom-Poisson superalgebra $A_{a}=$ $\left(A,{ }_{\alpha},[\cdot, \cdot]_{\alpha}, \alpha\right)$ with the nonzero products

$$
\begin{gathered}
e_{1 \cdot \alpha} e_{1}=a e_{1}, \\
e_{2} \cdot{ }_{\alpha} e_{2}=a e_{1}, \\
e_{1} \cdot{ }_{\alpha} e_{\alpha}=a e_{2}, \\
{\left[e_{2}, e_{2}\right]_{\alpha}=2 a e_{1} .}
\end{gathered}
$$

It is not a Poisson superalgebra when $a \neq 0,1$.

Example 10. Let $A=A_{\overline{0}} \oplus A_{\overline{1}}$ be a 3-dimensional $\mathbb{Z}_{2^{-}}$ graded vector space, where $A_{\overline{0}}$ is generated by $e_{1}, e_{2}$ and $A_{\overline{1}}$ is generated by $e_{3}$ and the nonzero products are given by

$$
\begin{gathered}
e_{1} \cdot e_{2}=e_{1}, \\
e_{2} \cdot e_{2}=e_{2}, \\
e_{3} \cdot e_{2}=e_{3}, \\
{\left[e_{1}, e_{2}\right]=a e_{1} ;}
\end{gathered}
$$

then $(A, \cdot,[\cdot, \cdot])$ is a Poisson superalgebra. For any $a \in \mathbb{C}$, we consider the homomorphism $\alpha: A \rightarrow A$ defined by

$$
\begin{aligned}
& \alpha\left(e_{1}\right)=a e_{1}, \\
& \alpha\left(e_{2}\right)=e_{1}+e_{2} .
\end{aligned}
$$

By Theorem 8 , for any $a \in \mathbb{C}$, there is the corresponding Hom-Poisson superalgebra $A_{\alpha}=\left(A,{ }_{\alpha},[\cdot, \cdot]_{\alpha}, \alpha\right)$ with the nonzero products

$$
\begin{aligned}
e_{1} \cdot{ }_{\alpha} e_{2} & =a e_{1}, \\
e_{2} \cdot{ }_{\alpha} e_{2} & =e_{1}+e_{2}, \\
{\left[e_{1}, e_{2}\right]_{\alpha} } & =a e_{1} .
\end{aligned}
$$

It is not a Poisson superalgebra when $a \neq 0,1$.

We know Hom-Poisson algebras are closed under tensor products ([8] Theorem 2.9). Here we aim to consider it in detail in the superalgebra case.

Theorem 11. Let $\left(A_{i},{ }_{i},[\cdot, \cdot]_{i}, \alpha_{i}\right)$ be Hom-Poisson superalgebras for $i=1,2$, and let $A=A_{1} \otimes A_{2}$. Define the operations $\alpha: A \rightarrow A$ and $\cdot,[\cdot, \cdot]: A^{\otimes 2} \rightarrow A$ by

$$
\begin{aligned}
\alpha= & \alpha_{1} \otimes \alpha_{2}, \\
\left(x_{1} \otimes x_{2}\right) \cdot\left(y_{1} \otimes y_{2}\right)= & (-1)^{\left|x_{2}\right|\left|y_{1}\right|}\left(x_{1 \cdot 1} y_{1}\right) \\
& \otimes\left(x_{2} \cdot{ }_{2} y_{2}\right) \\
{\left[x_{1} \otimes x_{2}, y_{1} \otimes y_{2}\right]=} & (-1)^{\left|x_{2}\right|\left|y_{1}\right|}\left[x_{1}, y_{1}\right]_{1} \otimes\left(x_{2} \cdot{ }_{2} y_{2}\right) \\
& +(-1)^{\left|x_{2}\right|\left|y_{1}\right|}\left(x_{1} \cdot y_{1}\right) \\
& \otimes\left[x_{2}, y_{2}\right]_{2},
\end{aligned}
$$

for $x_{i}, y_{i} \in A_{i}$. Then $(A, \cdot,[\cdot, \cdot], \alpha)$ is a Hom-Poisson superalgebra.
Proof. The $(A, \cdot, \alpha)$ is a supercommutative Hom-associative superalgebra following from the supercommutativity and Hom-associativity of both $\cdot_{i}$. Also, the supercommutativity of the ${ }_{i}$ and the antisupersymmetry of the $[\cdot, \cdot]_{i}$ imply the antisupersymmetry of $[\cdot, \cdot]$. It remains to prove the HomJacobi superidentity and the Hom-Leibniz superidentity in $A$.

To simplify the typography, we abbreviate $\cdot_{1}, \cdot_{2}$, and $\cdot$ using juxtaposition and drop the subscripts in $[\cdot, \cdot]_{i}$ and $\alpha_{i}$. Pick $x=$ $x_{1} \otimes x_{2}, y=y_{1} \otimes y_{2}, z=z_{1} \otimes z_{2} \in A$. Then

$$
\begin{aligned}
(-1)^{|x||z|}[[x, y], \alpha(z)] \\
=(-1)^{s_{1}}\left[\left[x_{1}, y_{1}\right] \otimes\left(x_{2} y_{2}\right), \alpha_{1}\left(z_{1}\right) \otimes \alpha_{2}\left(z_{2}\right)\right] \\
\quad+(-1)^{s_{1}}\left[\left(x_{1} y_{1}\right) \otimes\left[x_{2}, y_{2}\right], \alpha_{1}\left(z_{1}\right) \otimes \alpha_{2}\left(z_{2}\right)\right] \\
=(-1)^{s_{2}}\left[\left[x_{1}, y_{1}\right], \alpha_{1}\left(z_{1}\right)\right] \otimes\left(x_{2} y_{2}\right) \alpha_{2}\left(z_{2}\right) \\
\quad+(-1)^{s_{2}}\left[x_{1}, y_{1}\right] \alpha_{1}\left(z_{1}\right) \otimes\left[x_{2} y_{2}, \alpha_{2}\left(z_{2}\right)\right] \\
\quad+(-1)^{s_{2}}\left[x_{1} y_{1}, \alpha_{1}\left(z_{1}\right)\right] \otimes\left[x_{2}, y_{2}\right] \alpha_{2}\left(z_{2}\right) \\
\quad+(-1)^{s_{2}}\left(x_{1} y_{1}\right) \alpha_{1}\left(z_{1}\right) \otimes\left[\left[x_{2}, y_{2}\right], \alpha_{2}\left(z_{2}\right)\right]
\end{aligned}
$$

where $s_{1}=\left|x_{1}\right|\left|z_{1}\right|+\left|x_{1}\right|\left|z_{2}\right|+\left|x_{2}\right|\left|z_{1}\right|+\left|x_{2}\right|\left|z_{2}\right|+\left|x_{2}\right|\left|y_{1}\right|$, $s_{2}=\left|x_{1}\right|\left|z_{1}\right|+\left|x_{1}\right|\left|z_{2}\right|+\left|x_{2}\right|\left|z_{2}\right|+\left|x_{2}\right|\left|y_{1}\right|+\left|y_{2}\right|\left|z_{1}\right|$. Consider

$$
\begin{aligned}
(-1)^{|x \| y|}[[y, z], \alpha(x)] \\
=(-1)^{s_{3}}\left[\left[y_{1}, z_{1}\right], \alpha_{1}\left(x_{1}\right)\right] \otimes\left(y_{2} z_{2}\right) \alpha_{2}\left(x_{2}\right) \\
\quad+(-1)^{s_{3}}\left[y_{1}, z_{1}\right] \alpha_{1}\left(x_{1}\right) \otimes\left[y_{2} z_{2}, \alpha_{2}\left(x_{2}\right)\right] \\
\quad+(-1)^{s_{3}}\left[y_{1} z_{1}, \alpha_{1}\left(x_{1}\right)\right] \otimes\left[y_{2}, z_{2}\right] \alpha_{2}\left(x_{2}\right) \\
\quad+(-1)^{s_{3}}\left(y_{1} z_{1}\right) \alpha_{1}\left(x_{1}\right) \otimes\left[\left[y_{2}, z_{2}\right], \alpha_{2}\left(x_{2}\right)\right],
\end{aligned}
$$

where $s_{3}=\left|y_{1}\right|\left|x_{1}\right|+\left|y_{1}\right|\left|x_{2}\right|+\left|y_{2}\right|\left|x_{2}\right|+\left|y_{2}\right|\left|z_{1}\right|+\left|z_{2}\right|\left|x_{1}\right|$. Consider

$$
\begin{aligned}
(-1)^{|y||z|}[[z, x], \alpha(y)] \\
=(-1)^{s_{4}}\left[\left[z_{1}, x_{1}\right], \alpha_{1}\left(y_{1}\right)\right] \otimes\left(z_{2} x_{2}\right) \alpha_{2}\left(y_{2}\right) \\
\quad+(-1)^{s_{4}}\left[z_{1}, x_{1}\right] \alpha_{1}\left(y_{1}\right) \otimes\left[z_{2} x_{2}, \alpha_{2}\left(y_{2}\right)\right] \\
\quad+(-1)^{s_{4}}\left[z_{1} x_{1}, \alpha_{1}\left(y_{1}\right)\right] \otimes\left[z_{2}, x_{2}\right] \alpha_{2}\left(y_{2}\right) \\
\quad+(-1)^{s_{4}}\left(z_{1} x_{1}\right) \alpha_{1}\left(y_{1}\right) \otimes\left[\left[z_{2}, x_{2}\right], \alpha_{2}\left(y_{2}\right)\right],
\end{aligned}
$$

where $s_{4}=\left|z_{1}\right|\left|y_{1}\right|+\left|z_{1}\right|\left|y_{2}\right|+\left|z_{2}\right|\left|y_{2}\right|+\left|z_{2}\right|\left|x_{1}\right|+$ $\left|x_{2}\right|\left|y_{1}\right|$. Using the Hom-Jacobi superidentity in $A_{1}$ and the supercommutativity and Hom-associativity in $A_{2}$, we have

$$
\begin{aligned}
& (-1)^{s_{2}}\left[\left[x_{1}, y_{1}\right], \alpha_{1}\left(z_{1}\right)\right] \otimes\left(x_{2} y_{2}\right) \alpha_{2}\left(z_{2}\right) \\
& \quad+(-1)^{s_{3}}\left[\left[y_{1}, z_{1}\right], \alpha_{1}\left(x_{1}\right)\right] \otimes\left(y_{2} z_{2}\right) \alpha_{2}\left(x_{2}\right) \\
& \quad+(-1)^{s_{4}}\left[\left[z_{1}, x_{1}\right], \alpha_{1}\left(y_{1}\right)\right] \otimes\left(z_{2} x_{2}\right) \alpha_{2}\left(y_{2}\right) \\
& \quad=0 .
\end{aligned}
$$


Likewise, using the supercommutativity and Hom-associativity in $A_{1}$ and the Hom-Jacobi superidentity in $A_{2}$, we obtain

$$
\begin{aligned}
& (-1)^{s_{2}}\left(x_{1} y_{1}\right) \alpha_{1}\left(z_{1}\right) \otimes\left[\left[x_{2}, y_{2}\right], \alpha_{2}\left(z_{2}\right)\right] \\
& \quad+(-1)^{s_{3}}\left(y_{1} z_{1}\right) \alpha_{1}\left(x_{1}\right) \otimes\left[\left[y_{2}, z_{2}\right], \alpha_{2}\left(x_{2}\right)\right] \\
& \quad+(-1)^{s_{4}}\left(z_{1} x_{1}\right) \alpha_{1}\left(y_{1}\right) \otimes\left[\left[z_{2}, x_{2}\right], \alpha_{2}\left(y_{2}\right)\right] \\
& =0 .
\end{aligned}
$$

Using the Hom-Leibniz superidentity in $A_{i}$, then we have

$(-1)^{s_{2}}\left[x_{1}, y_{1}\right] \alpha_{1}\left(z_{1}\right) \otimes\left[x_{2} y_{2}, \alpha_{2}\left(z_{2}\right)\right]+(-1)^{s_{2}}$

$\cdot\left[x_{1} y_{1}, \alpha_{1}\left(z_{1}\right)\right] \otimes\left[x_{2}, y_{2}\right] \alpha_{2}\left(z_{2}\right)+(-1)^{s_{3}}\left[y_{1}, z_{1}\right]$

$\cdot \alpha_{1}\left(x_{1}\right) \otimes\left[y_{2} z_{2}, \alpha_{2}\left(x_{2}\right)\right]+(-1)^{s_{3}}\left[y_{1} z_{1}, \alpha_{1}\left(x_{1}\right)\right]$

$\otimes\left[y_{2}, z_{2}\right] \alpha_{2}\left(x_{2}\right)+(-1)^{s_{4}}\left[z_{1}, x_{1}\right] \alpha_{1}\left(y_{1}\right)$

$\otimes\left[z_{2} x_{2}, \alpha_{2}\left(y_{2}\right)\right]+(-1)^{s_{4}}\left[z_{1} x_{1}, \alpha_{1}\left(y_{1}\right)\right]$

$\otimes\left[z_{2}, x_{2}\right] \alpha_{2}\left(y_{2}\right)=(-1)^{s_{2}+\left|y_{2}\right|\left|z_{2}\right|}\left[x_{1}, y_{1}\right] \alpha_{1}\left(z_{1}\right)$

$\otimes\left[x_{2},\left(z_{2}\right)\right] \alpha_{2}\left(y_{2}\right)+(-1)^{s_{2}}\left[x_{1}, y_{1}\right] \alpha_{1}\left(z_{1}\right)$

$\otimes \alpha_{2}\left(x_{2}\right)\left[y_{2}, z_{2}\right]+(-1)^{s_{3}+\left|z_{2}\right|\left|x_{2}\right|}\left[y_{1}, z_{1}\right] \alpha_{1}\left(x_{1}\right)$

$\otimes\left[y_{2}, x_{2}\right] \alpha_{2}\left(z_{2}\right)+(-1)^{s_{3}}\left[y_{1}, z_{1}\right] \alpha_{1}\left(x_{1}\right)$

$\otimes \alpha_{2}\left(y_{2}\right)\left[z_{2}, x_{2}\right]+(-1)^{s_{3}+\left|z_{1}\right|\left|x_{1}\right|}\left[y_{1}, x_{1}\right] \alpha_{1}\left(z_{1}\right)$

$\otimes\left[y_{2}, z_{2}\right] \alpha_{2}\left(x_{2}\right)+(-1)^{s_{3}} \alpha_{1}\left(y_{1}\right)\left[z_{1}, x_{1}\right]$

$\otimes\left[y_{2}, z_{2}\right] \alpha_{2}\left(x_{2}\right)+(-1)^{s_{4}+\left|y_{2}\right|\left|z_{2}\right|}\left[z_{1}, x_{1}\right] \alpha_{1}\left(y_{1}\right)$

$\otimes\left[z_{2} x_{2}, \alpha_{2}\left(y_{2}\right)\right]+(-1)^{s_{4}}\left[z_{1}, x_{1}\right] \alpha_{1}\left(y_{1}\right)$

$\otimes\left[z_{2}, y_{2}\right] \alpha_{2}\left(x_{2}\right)+(-1)^{s_{4}}\left[z_{1}, x_{1}\right] \alpha_{1}\left(y_{1}\right)$

$\otimes \alpha_{2}\left(z_{2}\right)\left[x_{2}, y_{2}\right]+(-1)^{s_{4}+\left|x_{1}\right|\left|y_{1}\right|}\left[z_{1}, y_{1}\right] \alpha_{1}\left(x_{1}\right)$

$\otimes\left[z_{2}, x_{2}\right] \alpha_{2}\left(y_{2}\right)+(-1)^{s_{4}} \alpha_{1}\left(z_{1}\right)\left[x_{1}, y_{1}\right]$

$\otimes\left[z_{2}, x_{2}\right] \alpha_{2}\left(y_{2}\right)=\left\{(-1)^{s_{2}+\left|y_{2}\right|\left|z_{2}\right|}\left[x_{1}, y_{1}\right] \alpha_{1}\left(z_{1}\right)\right.$

$\otimes\left[x_{2}, z_{2}\right] \alpha_{2}\left(y_{2}\right)+(-1)^{s_{4}} \alpha_{1}\left(z_{1}\right)\left[x_{1}, y_{1}\right]$

$\left.\otimes\left[z_{2}, x_{2}\right] \alpha_{2}\left(y_{2}\right)\right\}+\left\{(-1)^{s_{2}}\left[x_{1}, y_{1}\right] \alpha_{1}\left(z_{1}\right)\right.$

$\otimes \alpha_{2}\left(x_{2}\right)\left[y_{2}, z_{2}\right]+(-1)^{s_{3}+\left|z_{1}\right|\left|x_{1}\right|}\left[y_{1}, x_{1}\right] \alpha_{1}\left(z_{1}\right)$

$\left.\otimes\left[y_{2}, z_{2}\right] \alpha_{2}\left(x_{2}\right)\right\}+\left\{(-1)^{s_{3}}\left[y_{1}, z_{1}\right] \alpha_{1}\left(x_{1}\right)\right.$

$\otimes \alpha_{2}\left(y_{2}\right)\left[z_{2}, x_{2}\right]+(-1)^{s_{3}+\left|z_{1}\right|\left|x_{1}\right|}\left[y_{1}, x_{1}\right] \alpha_{1}\left(z_{1}\right)$

$\left.\otimes\left[y_{2}, z_{2}\right] \alpha_{2}\left(x_{2}\right)\right\}+\left\{(-1)^{s_{3}}\left[y_{1}, z_{1}\right] \alpha_{1}\left(x_{1}\right)\right.$

$\otimes \alpha_{2}\left(y_{2}\right)\left[z_{2}, x_{2}\right]+(-1)^{s_{4}+\left|x_{1}\right|\left|y_{1}\right|}\left[z_{1}, y_{1}\right] \alpha_{1}\left(x_{1}\right)$

$\left.\otimes\left[z_{2}, x_{2}\right] \alpha_{2}\left(y_{2}\right)\right\}+\left\{(-1)^{s_{3}} \alpha_{1}\left(y_{1}\right)\left[z_{1}, x_{1}\right]\right.$

$$
\begin{aligned}
& \otimes\left[y_{2}, z_{2}\right] \alpha_{2}\left(x_{2}\right)+(-1)^{s_{4}+\left|x_{2}\right|\left|y_{2}\right|}\left[z_{1}, x_{1}\right] \alpha_{1}\left(y_{1}\right) \\
& \left.\otimes\left[z_{2}, y_{2}\right] \alpha_{2}\left(x_{2}\right)\right\}+\left\{(-1)^{s_{2}+\left|y_{1}\right|\left|z_{1}\right|}\left[x_{1}, z_{1}\right] \alpha_{1}\left(y_{1}\right)\right. \\
& \otimes\left[x_{2}, y_{2}\right] \alpha_{2}\left(z_{2}\right)+(-1)^{s_{4}}\left[z_{1}, x_{1}\right] \alpha_{1}\left(y_{1}\right) \\
& \left.\otimes \alpha_{2}\left(z_{2}\right)\left[x_{2}, y_{2}\right]\right\}=0+0+0+0+0+0=0 .
\end{aligned}
$$

This shows that $(A,[\cdot, \cdot], \alpha)$ satisfies the Hom-Jacobi superidentity:

$$
\begin{gathered}
(-1)^{|x||z|}[[x, y], \alpha(z)]+(-1)^{|x||y|}[[y, z], \alpha(x)] \\
+(-1)^{|y||z|}[[z, x], \alpha(y)]=0 .
\end{gathered}
$$

Finally, we check the Hom-Leibniz superidentity in A. Using the Hom-associativity and the Hom-Leibniz superidentity in the $A_{i}$, we have

$$
\begin{aligned}
& {[x y, \alpha(z)]=\left[\left(x_{1} \otimes y_{1}\right)\left(y_{1} \otimes y_{2}\right), \alpha_{1}\left(z_{1}\right) \otimes \alpha_{2}\left(z_{2}\right)\right]} \\
& =(-1)^{\left|x_{2}\right|\left|y_{1}\right|+\left|x_{2}\right|\left|z_{1}\right|+\left|y_{2}\right|\left|z_{1}\right|}\left[x_{1} y_{1}, \alpha_{1}\left(z_{1}\right)\right] \otimes\left(x_{2} y_{2}\right) \\
& \cdot \alpha_{2}\left(z_{2}\right)+(-1)^{\left|x_{2}\right|\left|y_{1}\right|+\left|x_{2}\right|\left|z_{1}\right|+\left|y_{2}\right|\left|z_{1}\right|}\left(x_{1} y_{1}\right) \alpha_{1}\left(z_{1}\right) \\
& \otimes\left[x_{2} y_{2}, \alpha_{2}\left(z_{2}\right)\right]=(-1)^{\left|x_{2} \| y_{1}\right|+\left|x_{2}\right|\left|z_{1}\right|+\left|y_{2}\right|\left|z_{1}\right|+\left|y_{1}\right|\left|z_{1}\right|} \\
& \cdot\left[x_{1}, z_{1}\right] \alpha_{1}\left(y_{1}\right) \otimes\left(x_{2} y_{2}\right) \alpha_{2}\left(z_{2}\right) \\
& +(-1)^{\left|x_{2}\right|\left|y_{1}\right|+\left|x_{2}\right|\left|z_{1}\right|+\left|y_{2}\right|\left|z_{1}\right|} \alpha_{1}\left(x_{1}\right)\left[y_{1}, z_{1}\right] \otimes\left(x_{2} y_{2}\right) \\
& \cdot \alpha_{2}\left(z_{2}\right)+(-1)^{\left|x_{2}\right|\left|y_{1}\right|+\left|x_{2}\right|\left|z_{1}\right|+\left|y_{2}\right|\left|z_{1}\right|+\left|y_{2}\right|\left|z_{2}\right|}\left(x_{1} y_{1}\right) \\
& \cdot \alpha_{1}\left(z_{1}\right) \otimes\left[x_{2}, z_{2}\right] \alpha_{2}\left(y_{2}\right) \\
& +(-1)^{\left|x_{2}\right|\left|y_{1}\right|+\left|x_{2}\right|\left|z_{1}\right|+\left|y_{2}\right|\left|z_{1}\right|}\left(x_{1} y_{1}\right) \alpha_{1}\left(z_{1}\right) \otimes \alpha_{2}\left(x_{2}\right) \\
& \cdot\left[y_{2}, z_{2}\right] \text {, } \\
& (-1)^{|y||z|}[x, z] \alpha(y)+\alpha(x)[y, z] \\
& =(-1)^{\left|y_{1}\right|\left|z_{1}\right|+\left|y_{1}\right|\left|z_{2}\right|+\left|y_{2}\right|\left|z_{1}\right|+\left|y_{2}\right|\left|z_{2}\right|+\left|x_{2}\right|\left|z_{1}\right|} \\
& \cdot\left(\left[x_{1}, z_{1}\right] \otimes\left(x_{2} z_{2}\right)\right)\left(\alpha_{1}\left(y_{1}\right) \otimes \alpha_{2}\left(y_{2}\right)\right) \\
& +(-1)^{\left|y_{1}\right|\left|z_{1}\right|+\left|y_{1}\right|\left|z_{2}\right|+\left|y_{2}\right|\left|z_{1}\right|+\left|y_{2}\right|\left|z_{2}\right|+\left|x_{2}\right|\left|z_{1}\right|} \\
& \cdot\left(\left(x_{1} z_{1}\right) \otimes\left[x_{2}, z_{2}\right]\right)\left(\alpha_{1}\left(y_{1}\right) \otimes \alpha_{2}\left(y_{2}\right)\right) \\
& +(-1)^{\left|y_{2}\right|\left|z_{1}\right|} \alpha_{1}\left(x_{1} \otimes \alpha_{2}\left(x_{2}\right)\right)\left(\left[y_{1}, z_{1}\right] \otimes\left(y_{2} z_{2}\right)\right) \\
& +(-1)^{\left|y_{2}\right|\left|z_{1}\right|} \alpha_{1}\left(x_{1} \otimes \alpha_{2}\left(x_{2}\right)\right)\left(\left(y_{1} z_{1}\right) \otimes\left[y_{2}, z_{2}\right]\right) \\
& =(-1)^{\left|y_{1}\right|\left|z_{1}\right|+\left|y_{2}\right|\left|z_{1}\right|+\left|x_{2}\right|\left|z_{1}\right|+\left|x_{2}\right|\left|y_{1}\right|}\left[x_{1}, z_{1}\right] \alpha_{1}\left(y_{1}\right) \\
& \otimes\left(x_{2} z_{2}\right) \alpha_{2}\left(y_{2}\right)+(-1)^{\left|y_{2}\right|\left|z_{1}\right|+\left|y_{2}\right|\left|z_{2}\right|+\left|x_{2}\right|\left|z_{1}\right|+\left|x_{2}\right|\left|y_{1}\right|} \\
& \cdot\left(x_{1} z_{1}\right) \alpha_{1}\left(y_{1}\right) \otimes\left[x_{2}, z_{2}\right] \alpha_{2}\left(y_{2}\right) \\
& +(-1)^{\left|y_{2}\right|\left|z_{1}\right|+\left|x_{2}\right|\left|y_{1}\right|+\left|x_{2}\right|\left|z_{1}\right|} \alpha_{1}\left(x_{1}\right)\left[y_{1}, z_{1}\right] \otimes \alpha_{2}\left(x_{2}\right)
\end{aligned}
$$




$$
\begin{aligned}
& \cdot\left(y_{2} z_{2}\right)+(-1)^{\left|y_{2}\right|\left|z_{1}\right|+\left|x_{2}\right|\left|y_{1}\right|+\left|x_{2}\right|\left|z_{1}\right|} \alpha_{1}\left(x_{1}\right)\left(y_{1} z_{1}\right) \\
& \otimes \alpha_{2}\left(x_{2}\right)\left[y_{2}, z_{2}\right]
\end{aligned}
$$

Therefore, we have

$$
[x y, \alpha(z)]=(-1)^{|y| z \mid}[x, z] \alpha(y)+\alpha(x)[y, z] .
$$

Setting $\alpha_{i}=I d_{A_{i}}$ in Theorem 11, we obtain the result about Poisson superalgebras.

Corollary 12. Let $\left(A_{i},{ }_{i},[\cdot, \cdot]_{i},\right)$ be Poisson superalgebras for $i=1,2$, and let $A=A_{1} \otimes A_{2}$. Define the operations $\cdot,[\cdot, \cdot]$ : $A^{\otimes 2} \rightarrow A$ by

$$
\begin{aligned}
\left(x_{1} \otimes x_{2}\right) \cdot\left(y_{1} \otimes y_{2}\right)= & (-1)^{\left|x_{2}\right|\left|y_{1}\right|}\left(x_{1 \cdot 1} y_{1}\right) \\
& \otimes\left(x_{2} \cdot{ }_{2} y_{2}\right), \\
{\left[x_{1} \otimes x_{2}, y_{1} \otimes y_{2}\right]=} & (-1)^{\left|x_{2}\right|\left|y_{1}\right|}\left[x_{1}, y_{1}\right]_{1} \otimes\left(x_{2} \cdot y_{2}\right) \\
& +(-1)^{\left|x_{2}\right|\left|y_{1}\right|}\left(x_{1 \cdot 1} y_{1}\right) \\
& \otimes\left[x_{2}, y_{2}\right]_{2},
\end{aligned}
$$

for $x_{i}, y_{i} \in A_{i}$. Then $(A, \cdot,[\cdot, \cdot])$ is a Poisson superalgebra.

\section{Admissible Hom-Poisson Superalgebras}

A Poisson algebra has two binary operations, the Lie bracket and the commutative associative product. It is shown in [10] that Poisson algebras can be described using only one binary operation via the polarization-depolarization process. Moreover, the result of Poisson algebras is extended to HomPoisson algebras in [8]. In other words, the paper shows that a Hom-Poisson algebra can be described using only the twisting map and one binary operation. The purpose of this section is to extend this alternative description of Poisson algebras to Hom-Poisson superalgebras.

Definition 13. An admissible Hom-Poisson superalgebra $A$ is a Hom-superalgebra satisfying

$$
\begin{aligned}
3 A(x, y, z)+(-1)^{|x||y|}(y x) \alpha(z) \\
-(-1)^{|y||z|}(x z) \alpha(y) \\
-(-1)^{|x||y|+|x||z|}(y z) \alpha(x) \\
+(-1)^{|x||z|+|y||z|}(z x) \alpha(y)=0,
\end{aligned}
$$

where $A(x, y, z)=(x y) \alpha(z)-\alpha(x)(y z)$, for any homogeneous elements $x, y, z \in A$, the identity (32) is called the HomRemm identity.

Remark 14. In particular, taking $\alpha=I d_{A}$, we find the notion of admissible Poisson superalgebra presented in [8].
Theorem 15. Let $(A, \cdot,[\cdot, \cdot], \alpha)$ be a double Hom-superalgebra. Then $(A, \cdot,[\cdot, \cdot], \alpha)$ is a Hom-Poisson superalgebra if and only if there exists on $A$ a nonassociative product $x y$ such that $(A, \cdot, \alpha)$ is an admissible Hom-Poisson superalgebra.

Proof. Assume that $(A, \cdot,[\cdot, \cdot], \alpha)$ is a Hom-Poisson superalgebra. Consider the multiplication

$$
x y=x \cdot y+[x, y] .
$$

We deduce that

$$
x \cdot y=\frac{1}{2}\left(x y+(-1)^{|x||y|} y x\right) .
$$

Thus the associativity condition can be denoted by

$$
\begin{aligned}
v_{1}(x, y, z)= & A(x, y, z) \\
& -(-1)^{|x||y|+|x||z|+|y||z|} A(z, y, x) \\
& +(-1)^{|x||y|}(y x) \alpha(z) \\
& -(-1)^{|y||z|} \alpha(x)(z y) \\
& -(-1)^{|x||y|+|x||z|}(y z) \alpha(x) \\
& +(-1)^{|x||z|+|y||z|} \alpha(z)(x y)=0,
\end{aligned}
$$

where $A(x, y, z)=(x y) \alpha(z)-\alpha(x)(y z)$. Likewise, the HomPoisson bracket can be denoted by

$$
[x, y]=\frac{1}{2}\left(x y-(-1)^{|x||y|} y x\right)
$$

and the Hom-super Jacobi condition

$$
\begin{aligned}
v_{2}(x, y, z)= & (-1)^{|x| z \mid} A(x, y, z) \\
& -(-1)^{|x||y|+|x||z|} A(y, x, z) \\
& -(-1)^{|x||y|+|y||z|} A(z, y, x) \\
& -(-1)^{|x||z|+|y||z|} A(x, z, y) \\
& +(-1)^{|x||y|} A(y, z, x) \\
& +(-1)^{|y||z|} A(z, x, y)=0 .
\end{aligned}
$$

The Hom-Leibniz superidentity can be denoted by

$$
\begin{aligned}
v_{3}(x, y, z)= & A(x, y, z)-(-1)^{|x||y|} A(y, x, z) \\
& +(-1)^{|x||y|+|x||z|+|y||z|} A(z, y, x) \\
& +(-1)^{|y||z|} A(x, z, y) \\
& +(-1)^{|x||y|+|x||z|} A(y, z, x) \\
& -(-1)^{|x||z|+|y||z|} A(z, x, y)=0 .
\end{aligned}
$$


Let us consider the vector

$$
\begin{aligned}
& v(x, y, z)=\frac{1}{3}\left\{(-1)^{|x||y|}(y x) \alpha(z)\right. \\
& -(-1)^{|y||z|}(x z) \alpha(y)-(-1)^{|x||y|+|x||z|}(y z) \alpha(x) \\
& \left.+(-1)^{|x||z|+|y||z|}(z x) \alpha(y)\right\}+(x y) \alpha(z)-\alpha(x) \\
& \quad \cdot(y z) .
\end{aligned}
$$

Then

$$
\begin{aligned}
& v(x, y, z)=\frac{1}{6}\left\{2 v_{1}(x, y, z)+(-1)^{|x||z|} v_{2}(x, y, z)\right. \\
& \left.+v_{3}(x, y, z)+2(-1)^{|x||z|+|y||z|} v_{3}(z, x, y)\right\} .
\end{aligned}
$$

We deduce that the product $x y$ satisfies

$$
v(x, y, z)=0
$$

for any homogeneous elements $x, y, z \in A$.

Conversely, assume that the produce of the nonassociative product $A$ satisfies $v(x, y, z)=0$ for any homogeneous elements $x, y, z \in A$. Let $v_{1}(x, y, z), v_{2}(x, y, z), v_{3}(x, y, z)$ be the elements of $A$ defined in the first part, respectively, in relation to the Hom-associativity, Hom-super Jacobi, and Hom-super Leibniz relations. We have

$$
\begin{aligned}
& v_{1}(x, y, z)=v(x, y, z) \\
& -(-1)^{|x||y|+|x||z|+|y||z|} v(z, y, x) \\
& +(-1)^{|y||z|} v(x, z, y) \\
& -(-1)^{|x||z|+|y||z|} v(z, x, y) \\
& v_{2}(x, y, z)=(-1)^{|x||z|} v(x, y, z) \\
& -(-1)^{|x||y|+|x||z|} v(y, x, z) \\
& -(-1)^{|x||y|+|y||z|} v(z, y, x) \\
& -(-1)^{|x||z|+|y||z|} v(x, z, y) \\
& +(-1)^{|x||y|} v(y, z, x) \\
& +(-1)^{|y||z|} v(z, x, y) \text {, } \\
& v_{3}(x, y, z)=v(x, y, z)-(-1)^{|x||y|} v(y, x, z) \\
& +(-1)^{|x||y|+|x||z|+|y||z|} v(z, y, x) \\
& +(-1)^{|y||z|} v(x, z, y) \\
& +(-1)^{|x||y|+|x||z|} v(y, z, x) \\
& -(-1)^{|x||z|+|y||z|} v(z, x, y) \text {. }
\end{aligned}
$$

Taking $\alpha=I d_{A}$ in Theorem 15 , we obtain the following result, which is Theorem 1 in [7].
Corollary 16. Let $(A, \cdot,[\cdot, \cdot])$ be a double superalgebra. Then $(A, \cdot,[\cdot, \cdot])$ is a Poisson superalgebra if and only if there exists on $A$ a nonassociative product $x y$ satisfying

$$
\begin{aligned}
3 A & (x, y, z)+(-1)^{|x||y|}(y x) z-(-1)^{|y||z|}(x z) y \\
& -(-1)^{|x||y|+|x||z|}(y z) x+(-1)^{|x||z|+|y||z|}(z x) y \\
& =0,
\end{aligned}
$$

where $A(x, y, z)=(x y) z-x(y z)$, for any homogeneous elements $x, y, z \in A$.

Definition 17. A Hom-nonassociative superalgebra $(A, \cdot, \alpha)$ is called Hom-superflexive if the multiplication $x y$ satisfies

$$
A(x, y, z)+(-1)^{|x||y|+|x||z|+|y||z|} A(z, y, x)=0
$$

for any homogeneous elements $x, y, z \in A$, where $A_{\alpha}(x, y$, $z)=(x y) \alpha(z)-\alpha(x)(y z)$ is called a Hom-associator of the multiplication.

Proposition 18. Let $(A, \cdot,[\cdot, \cdot], \alpha)$ be a Hom-Poisson superalgebra. Then the Hom-Remm product defining the Hom-Poisson superalgebra structure is Hom-superflexive.

Proof. Let

$$
\begin{aligned}
B & (x, y, z) \\
& =3\left\{A(x, y, z)+(-1)^{|x||y|+|x||z|+|y||z|} A(z, y, x)\right\} .
\end{aligned}
$$

We have

$$
\begin{aligned}
B( & , y, z)=-(-1)^{|x||y|}(y x) \alpha(z)+(-1)^{|y||z|}(x z) \\
& \cdot \alpha(y)+(-1)^{|x||y|+|x||z|}(y z) \alpha(x)-(-1)^{|x||z|+|y||z|} \\
& \cdot(z x) \alpha(y)+(-1)^{|x||y|+|x||z|+|y||z|} \\
& \cdot\left\{-(-1)^{|z||y|}(y z) \alpha(x)\right. \\
& +(-1)^{|y||x|}(z x) \alpha(y)+(-1)^{|z||y|+|z||x|}(y x) \alpha(y) \\
& \left.-(-1)^{|z||x|+|y \|| z \mid}(x z) \alpha(y)\right\}=\left\{-(-1)^{|x||y|}\right. \\
& \left.+(-1)^{|x||y|}\right\}(y x) \alpha(z)+\left\{(-1)^{|y||z|}-(-1)^{|y||z|}\right\} \\
& \cdot(x z) \alpha(y)+\left\{(-1)^{|x||y|+|x||z|}-(-1)^{|x||y|+|x||z|}\right\} \\
& \cdot(y z) \alpha(x)+\left\{-(-1)^{|x||z|+|y||z|}+(-1)^{|x||z|+|y||z|}\right\} \\
& \cdot(z x) \alpha(y)=0 .
\end{aligned}
$$

Taking $\alpha=I d_{A}$ in Proposition 18, we obtain the following result, which is Proposition 3 in [7].

Corollary 19. Let $(A, \cdot,[\cdot, \cdot])$ be a Poisson superalgebra. Then the Remm product defining Poisson superalgebra structure is superflexive. 
Remark 20. The deformation cohomology of Hom-Poisson superalgebras can be computed with the Hom-Remm identity, which is similar to the method in [11]. This content is not primary in the paper, we do not have a detailed discussion here.

\section{A Classification of 2-Dimensional Hom-Poisson Superalgebras}

In this section, we only consider that $A_{\overline{1}}$ is nontrivial. $A_{2}^{i}$ denotes one of the 2-dimensional admissible Poisson superalgebra types. $\alpha_{2}^{i}$ denotes one of the homomorphism types corresponding to $A_{2}^{i} . \widetilde{A}_{2}^{i}$ denotes one of the 2-dimensional admissible Hom-Poisson superalgebra types corresponding to $\alpha_{2}^{i}$. In the following, the products equal to zero are omitted.

Lemma 21. Let $(A,())$ be an admissible Poisson superalgebra and let $\alpha: A \rightarrow A$ be an even Poisson superalgebra endomorphism. Then $\left(A,()_{\alpha}, \alpha\right)$ is an admissible Hom-Poisson superalgebra, where $(x y)_{\alpha}=\alpha(x y)$.

Proof. It is straightforward by Definition 13.

Lemma 22 (see [7]). Let $(A,())$ be a 2-dimensional admissible Poisson superalgebra with a basis $\left\{e_{0}, e_{1}\right\}$, where $e_{0} \in A_{\overline{0}}, e_{1} \in$ $A_{\overline{1}}$. Then $A$ is one of the following types:

$$
\begin{aligned}
& A_{2}^{1}: e_{0} e_{0}=a e_{0}, e_{0} e_{1}=a e_{1}, e_{1} e_{0}=a e_{1}, e_{1} e_{1}=d e_{0}, \\
& d \neq 0 . \\
& A_{2}^{2}: e_{0} e_{0}=a e_{0} . \\
& A_{2}^{3}: e_{0} e_{0}=a e_{0}, e_{0} e_{1}=a e_{1}, e_{1} e_{0}=a e_{1}, a \neq 0 . \\
& A_{2}^{4}: e_{0} e_{1}=b e_{1}, e_{1} e_{0}=-b e_{1}, b \neq 0 .
\end{aligned}
$$

Proof. Let

$$
\begin{aligned}
& e_{0} e_{0}=a e_{0}, \\
& e_{0} e_{1}=b e_{1}, \\
& e_{1} e_{0}=c e_{1}, \\
& e_{1} e_{1}=d e_{0} .
\end{aligned}
$$

By Corollary 16, we have

$$
\begin{aligned}
3(a-b) b+a b-2 b c+c^{2} & =0, \\
d(b-a) & =0, \\
3(a-c) c+a b-2 b c+c^{2} & =0, \\
d(3 c-b-2 a) & =0, \\
d(b-c) & =0 .
\end{aligned}
$$

Now we consider the cases as follows.

Case 1 . If $d \neq 0$, then $b=a=c$; hence we have $A_{2}^{1}$.

Case 2. If $d=0$, then $(b-c)(a-b-c)=0$.
Subcase 2.1. If $b=c$, then $b(a-b)=0$.

If $b=0$, then we have $A_{2}^{2}$.

If $b \neq 0$, then $a=b=c \neq 0$; hence we have $A_{2}^{3}$.

Subcase 2.2. If $b \neq c$, then $b=-c \neq 0, a=0$; hence we have $A_{2}^{4}$.

Remark 23. Lemma 5.2 in [6] has been obtained; however, there is one minor inaccuracy on the product operation in that proof.

Lemma 24. Let $(A,())$ be a 2-dimensional admissible Poisson superalgebra with $\operatorname{dim} A_{\overline{0}}=1$ and $\operatorname{dim} A_{\overline{1}}=1$. Then an even homomorphism $\alpha$ of type $A_{2}^{1}$ is as follows:

$$
\begin{gathered}
\alpha_{2(1)}^{1}:\left\{\begin{array}{l}
\alpha\left(e_{0}\right)=k^{2} e_{0} \\
\alpha\left(e_{1}\right)=k e_{1} ;
\end{array}\right. \\
\alpha_{2(2)}^{1}:\left\{\begin{array}{l}
\alpha\left(e_{0}\right)=0 \\
\alpha\left(e_{1}\right)=0 ;
\end{array}\right. \\
\alpha_{2(3)}^{1}:\left\{\begin{array}{l}
\alpha\left(e_{0}\right)=e_{0} \\
\alpha\left(e_{1}\right)= \pm e_{1} .
\end{array}\right.
\end{gathered}
$$

Proof. Let

$$
\begin{aligned}
& \alpha\left(e_{0}\right)=a_{10} e_{0}, \\
& \alpha\left(e_{1}\right)=a_{11} e_{1} .
\end{aligned}
$$

From $\alpha$ is an even homomorphism, we obtain

$$
\begin{aligned}
& \alpha\left(e_{0} e_{0}\right)=\alpha\left(e_{0}\right) \alpha\left(e_{0}\right), \\
& \alpha\left(e_{0} e_{1}\right)=\alpha\left(e_{0}\right) \alpha\left(e_{1}\right), \\
& \alpha\left(e_{1} e_{0}\right)=\alpha\left(e_{1}\right) \alpha\left(e_{0}\right), \\
& \alpha\left(e_{1} e_{1}\right)=\alpha\left(e_{1}\right) \alpha\left(e_{1}\right) .
\end{aligned}
$$

By Lemma 22 and (50) and (51), we obtain

$$
\begin{aligned}
a a_{10}\left(a_{10}-1\right) & =0, \\
a a_{11}\left(a_{10}-1\right) & =0, \\
a_{10} & =a_{11}^{2} .
\end{aligned}
$$

Case 1. If $a=0$, then we have $\alpha_{2(1)}^{1}$.

Case 2. If $a \neq 0$, then we consider two cases as follows.

$$
\begin{aligned}
& \text { If } a_{10}=0 \text {, then } a_{11}=0 \text {; hence we have } \alpha_{2(2)}^{1} \text {. } \\
& \text { If } a_{10} \neq 0 \text {, then } a_{10}=1, a_{11}= \pm 1 \text {; hence we have } \alpha_{2(3)}^{1} .
\end{aligned}
$$

Corollary 25. Let $\left(\widetilde{A},()_{\alpha}, \alpha\right)$ be a 2-dimensional admissible Hom-Poisson superalgebra. Then $\widetilde{A}$ with respect to $\alpha_{2}^{1}$ is as follows:

$$
\begin{aligned}
& \widetilde{A}_{2(1)}:\left(e_{1} e_{1}\right)_{\alpha}=d k^{2} e_{0}, d \neq 0 . \\
& \widetilde{A}_{2(2)}:(A A)_{\alpha}=0 .
\end{aligned}
$$




$$
\widetilde{A}_{2(3)}:\left(e_{0} e_{0}\right)_{\alpha}=a e_{0},\left(e_{0} e_{1}\right)_{\alpha}= \pm a e_{1},\left(e_{1} e_{0}\right)_{\alpha}= \pm a e_{1} \text {, }
$$$$
\left(e_{1} e_{1}\right)_{\alpha}=d e_{0}, a \neq 0, d \neq 0 \text {. }
$$

Proof. Apply Lemmas 21, 22, and 24.

Lemma 26. Let $(A,())$ be a 2-dimensional admissible Poisson superalgebra with $\operatorname{dim} A_{\overline{0}}=1$ and $\operatorname{dim} A_{\overline{1}}=1$. Then an even homomorphism $\alpha$ of type $A_{2}^{2}$ is as follows:

$$
\begin{aligned}
& \alpha_{2(1)}^{2}:\left\{\begin{array}{l}
\alpha\left(e_{0}\right)=k_{0} e_{0} \\
\alpha\left(e_{1}\right)=k e_{1} ;
\end{array}\right. \\
& \alpha_{2(2)}^{2}:\left\{\begin{array}{l}
\alpha\left(e_{0}\right)=0 \\
\alpha\left(e_{1}\right)=k e_{1} ;
\end{array}\right. \\
& \alpha_{2(3)}^{2}:\left\{\begin{array}{l}
\alpha\left(e_{0}\right)=e_{0} \\
\alpha\left(e_{1}\right)=k e_{1} .
\end{array}\right.
\end{aligned}
$$

Proof. By Lemma 22 and (20) and (21), we obtain $a a_{10}\left(a_{10}-\right.$ 1) $=0$.

Case 1. Suppose that $a=0$, we have $\alpha_{2(1)}^{2}$.

Case 2. Suppose that $a \neq 0$, we consider two cases as follows.

If $a_{10}=0$, then we have $\alpha_{2(2)}^{2}$.

If $a_{10} \neq 0$, then $a_{10}=1$; hence we have $\alpha_{2(3)}^{2}$.

Corollary 27. Let $\left(\widetilde{A},()_{\alpha}, \alpha\right)$ be a 2-dimensional admissible Hom-Poisson superalgebra. Then $\widetilde{A}$ with respect to $\alpha_{2}^{2}$ is as follows:

$$
\begin{aligned}
& \widetilde{A}_{2(1)}^{2}:(A A)_{\alpha}=0 . \\
& \widetilde{A}_{2(2)}^{2}:(A A)_{\alpha}=0 . \\
& \widetilde{A}_{2(3)}^{2}:\left(e_{0} e_{0}\right)_{\alpha}=a e_{0}, a \neq 0 .
\end{aligned}
$$

Proof. Apply Lemmas 21, 22, and 26.

Lemma 28. Let $(A,())$ be a 2-dimensional admissible Poisson superalgebra with $\operatorname{dim} A_{\overline{0}}=1$ and $\operatorname{dim} A_{\overline{1}}=1$. Then an even homomorphism $\alpha$ of type $A_{2}^{3}$ is as follows:

$$
\begin{gathered}
\alpha_{2(1)}^{3}:\left\{\begin{array}{l}
\alpha\left(e_{0}\right)=0 \\
\alpha\left(e_{1}\right)=0 ;
\end{array}\right. \\
\alpha_{2(2)}^{3}:\left\{\begin{array}{l}
\alpha\left(e_{0}\right)=e_{0} \\
\alpha\left(e_{1}\right)=k e_{1} ;
\end{array}\right. \\
\alpha_{2(3)}^{3}:\left\{\begin{array}{l}
\alpha\left(e_{0}\right)=0 \\
\alpha\left(e_{1}\right)=0 ;
\end{array}\right.
\end{gathered}
$$$$
\alpha_{2(4)}^{3}:\left\{\begin{array}{l}
\alpha\left(e_{0}\right)=e_{0} \\
\alpha\left(e_{1}\right)=0 ;
\end{array}\right.
$$$$
\alpha_{2(5)}^{3}:\left\{\begin{array}{l}
\alpha\left(e_{0}\right)=e_{0} \\
\alpha\left(e_{1}\right)=k e_{1},
\end{array} \quad k \neq 0 .\right.
$$

Proof. By Lemma 22 and (20) and (21), we obtain

$$
\begin{aligned}
& a_{10}\left(a_{10}-1\right)=0, \\
& a_{11}\left(a_{10}-1\right)=0 .
\end{aligned}
$$

Case 1. If $a_{10}=0$, then $a_{11}=0$; hence we have $\alpha_{2(1)}^{3}$.

Case 2. If $a_{10} \neq 0$, then $a_{10}=1$; hence we have $\alpha_{2(2)}^{3}$.

Case 3. If $a_{11}=0$, then we consider two cases as follows.

If $a_{10}=0$, we have $\alpha_{2(3)}^{3}$.

If $a_{10} \neq 0$, then $a_{10}=1$; hence we have $\alpha_{2(4)}^{3}$.

Case 4. If $a_{11} \neq 0$, then $a_{10}=1$; hence we have $\alpha_{2(5)}^{3}$.

Corollary 29. Let $\left(\widetilde{A},()_{\alpha}, \alpha\right)$ be a 2-dimensional admissible Hom-Poisson superalgebra. Then $\widetilde{A}$ with respect to $\alpha_{2}^{3}$ is as follows:

$$
\begin{aligned}
& \widetilde{A}_{2(1)}^{3}:(A A)_{\alpha}=0 . \\
& \widetilde{A}_{2(2)}^{3}:\left(e_{0} e_{0}\right)_{\alpha}=a e_{0},\left(e_{0} e_{1}\right)_{\alpha}=k a e_{1},\left(e_{1} e_{0}\right)_{\alpha}=k a e_{1}, \\
& a \neq 0 . \\
& \widetilde{A}_{2(3)}^{3}:(A A)_{\alpha}=0 . \\
& \widetilde{A}_{2(4)}^{3}:\left(e_{0} e_{0}\right)_{\alpha}=a e_{0}, a \neq 0 . \\
& \widetilde{A}_{2(5)}^{3}:\left(e_{0} e_{0}\right)_{\alpha}=a e_{0},\left(e_{0} e_{1}\right)_{\alpha}=k a e_{1},\left(e_{1} e_{0}\right)_{\alpha}=k^{3} e_{1}, \\
& a \neq 0, k \neq 0 .
\end{aligned}
$$

Proof. Apply Lemmas 21, 22, and 28.

Lemma 30. Let $(A,())$ be a 2-dimensional admissible Poisson superalgebra with $\operatorname{dim} A_{\overline{0}}=1$ and $\operatorname{dim} A_{\overline{1}}=1$. Then an even homomorphism $\alpha$ of type $A_{2}^{4}$ is as follows:

$$
\begin{gathered}
\alpha_{2(1)}^{4}:\left\{\begin{array}{l}
\alpha\left(e_{0}\right)=k e_{0} \\
\alpha\left(e_{1}\right)=0 ;
\end{array}\right. \\
\alpha_{2(2)}^{4}:\left\{\begin{array}{l}
\alpha\left(e_{0}\right)=e_{0} \\
\alpha\left(e_{1}\right)=k e_{1},
\end{array}\right.
\end{gathered}
$$

Proof. By Lemma 22 and (20) and (21), we obtain $a_{11}\left(a_{10}-\right.$ 1) $=0$.

Case 1. If $a_{10}=0$, then we have $\alpha_{2(1)}^{4}$.

Case 2. If $a_{10} \neq 0$, then $a_{10}=1$; hence we have $\alpha_{2(2)}^{4}$. 
Corollary 31. Let $\left(\widetilde{A},()_{\alpha}, \alpha\right)$ be a 2-dimensional admissible Hom-Poisson superalgebra. Then $\widetilde{A}$ with respect to $\alpha_{2}^{4}$ is as follows:

$$
\begin{aligned}
& \widetilde{A}_{2(1)}^{4}:(A A)_{\alpha}=0 . \\
& \widetilde{A}_{2(2)}^{4}:\left(e_{0} e_{1}\right)_{\alpha}=k b e_{1},\left(e_{1} e_{0}\right)_{\alpha}=-k b e_{1}, b \neq 0, k \neq 0 .
\end{aligned}
$$

Proof. Apply Lemmas 21, 22, and 30.

Theorem 32. Let $(A,())$ be a 2-dimensional admissible Poisson superalgebra with $\operatorname{dim} A_{\overline{0}}=1$ and $\operatorname{dim} A_{\overline{1}}=1$. Then an even homomorphism $\alpha$ of $A$ is as follows:

$$
\begin{aligned}
& \alpha_{2(1)}^{1}:\left\{\begin{array}{l}
\alpha\left(e_{0}\right)=k^{2} e_{0} \\
\alpha\left(e_{1}\right)=k e_{1} ;
\end{array}\right. \\
& \alpha_{2(2)}^{1}:\left\{\begin{array}{l}
\alpha\left(e_{0}\right)=0 \\
\alpha\left(e_{1}\right)=0 ;
\end{array}\right. \\
& \alpha_{2(3)}^{1}:\left\{\begin{array}{l}
\alpha\left(e_{0}\right)=e_{0} \\
\alpha\left(e_{1}\right)= \pm e_{1} ;
\end{array}\right. \\
& \alpha_{2(1)}^{2}:\left\{\begin{array}{l}
\alpha\left(e_{0}\right)=k_{0} e_{0} \\
\alpha\left(e_{1}\right)=k e_{1} ;
\end{array}\right. \\
& \alpha_{2(2)}^{2}:\left\{\begin{array}{l}
\alpha\left(e_{0}\right)=0 \\
\alpha\left(e_{1}\right)=k e_{1} ;
\end{array}\right. \\
& \alpha_{2(3)}^{2}:\left\{\begin{array}{l}
\alpha\left(e_{0}\right)=e_{0} \\
\alpha\left(e_{1}\right)=k e_{1} ;
\end{array}\right. \\
& \alpha_{2(1)}^{3}:\left\{\begin{array}{l}
\alpha\left(e_{0}\right)=0 \\
\alpha\left(e_{1}\right)=0 ;
\end{array}\right. \\
& \alpha_{2(2)}^{3}:\left\{\begin{array}{l}
\alpha\left(e_{0}\right)=e_{0} \\
\alpha\left(e_{1}\right)=k e_{1} ;
\end{array}\right. \\
& \alpha_{2(3)}^{3}:\left\{\begin{array}{l}
\alpha\left(e_{0}\right)=0 \\
\alpha\left(e_{1}\right)=0 ;
\end{array}\right. \\
& \alpha_{2(4)}^{3}:\left\{\begin{array}{l}
\alpha\left(e_{0}\right)=e_{0} \\
\alpha\left(e_{1}\right)=0 ;
\end{array}\right. \\
& \alpha_{2(5)}^{3}:\left\{\begin{array}{l}
\alpha\left(e_{0}\right)=e_{0} \\
\alpha\left(e_{1}\right)=k e_{1}, \quad k \neq 0 ;
\end{array}\right.
\end{aligned}
$$

$$
\begin{gathered}
\alpha_{2(1)}^{4}:\left\{\begin{array}{l}
\alpha\left(e_{0}\right)=k e_{0} \\
\alpha\left(e_{1}\right)=0 ;
\end{array}\right. \\
\sigma_{2(2)}^{4}:\left\{\begin{array}{l}
\alpha\left(e_{0}\right)=e_{0} \\
\alpha\left(e_{1}\right)=k e_{1},
\end{array} \quad k \neq 0 .\right.
\end{gathered}
$$

Proof. Apply Lemmas 24-30.

Corollary 33. Let $\left(\widetilde{A},()_{\alpha}, \alpha\right)$ be a 2-dimensional admissible Hom-Poisson superalgebra. Then $\widetilde{A}$ with respect to $\alpha_{2}^{i}$ is as follows:

$$
\begin{aligned}
& \widetilde{A}_{2(1)}^{1}:\left(e_{1} e_{1}\right)_{\alpha}=d k^{2} e_{0}, d \neq 0 . \\
& \widetilde{A}_{2(2)}^{1}:(A A)_{\alpha}=0 . \\
& \widetilde{A}_{2(3)}^{1}:\left(e_{0} e_{0}\right)_{\alpha}=a e_{0},\left(e_{0} e_{1}\right)_{\alpha}= \pm a e_{1},\left(e_{1} e_{0}\right)_{\alpha}= \pm a e_{1}, \\
& \left(e_{1} e_{1}\right)_{\alpha}=d e_{0}, a \neq 0, d \neq 0 . \\
& \widetilde{A}_{2(1)}^{2}:(A A)_{\alpha}=0 . \\
& \widetilde{A}_{2(2)}^{2}:(A A)_{\alpha}=0 . \\
& \widetilde{A}_{2(3)}^{2}:\left(e_{0} e_{0}\right)_{\alpha}=a e_{0}, a \neq 0 . \\
& \widetilde{A}_{2(1)}^{3}:(A A)_{\alpha}=0 . \\
& \widetilde{A}_{2(2)}^{3}:\left(e_{0} e_{0}\right)_{\alpha}=a e_{0},\left(e_{0} e_{1}\right)_{\alpha}=k a e_{1},\left(e_{1} e_{0}\right)_{\alpha}=k a e_{1}, \\
& a \neq 0 . \\
& \widetilde{A}_{2(3)}^{3}:(A A)_{\alpha}=0 . \\
& \widetilde{A}_{2(4)}^{3}:\left(e_{0} e_{0}\right)_{\alpha}=a e_{0}, a \neq 0 . \\
& \widetilde{A}_{2(5)}^{3}:\left(e_{0} e_{0}\right)_{\alpha}=a e_{0},\left(e_{0} e_{1}\right)_{\alpha}=k a e_{1},\left(e_{1} e_{0}\right)_{\alpha}=k a e_{1}, \\
& a \neq 0, k \neq 0 . \\
& \widetilde{A}_{2(1)}^{4}:(A A)_{\alpha}=0 . \\
& \widetilde{A}_{2(2)}^{4}:\left(e_{0} e_{1}\right)_{\sigma}=k b e_{1},\left(e_{1} e_{0}\right)_{\alpha}=-k b e_{1}, b \neq 0, k \neq 0 .
\end{aligned}
$$

Proof. Apply Corollaries 25-31.

Remark 34. (1) Some nonisomorphic 2-dimensional admissible Poisson superalgebras have isomorphic admissible Hom-Poisson deformations. For example, the admissible Poisson superalgebras $A_{2}^{2}$ (with $k=0$ in its algebra homomorphism $\alpha_{2(3)}^{2}$ ) and $A_{2}^{3}$ can be deformed into isomorphic admissible Hom-Poisson superalgebras $\widetilde{A}_{2(3)}^{2}$ and $\widetilde{A}_{2(4)}^{3}$. There are several other such pairs in the cases above.

(2) We give a classification of 2-dimensional admissible Hom-Poisson superalgebras in Corollary 33. Using Theorem 15, a classification of 2-dimensional Hom-Poisson superalgebras will be obviously obtained.

\section{Conflict of Interests}

The authors declare that there is no conflict of interests regarding the publication of this paper. 


\section{Acknowledgment}

This work was supported by National Natural Science Foundation of China (no. 11471090) and the Natural Science Foundation of Jilin Province (no. 20130101068JC) and the Fundamental Research Funds for the Central Universities of China (no. 14QNJJ003).

\section{References}

[1] I. Vaisman, Lectures on the Geometry of Poisson Manifolds, Birkhäauser, Basel, Switzerland, 1994.

[2] V. G. Drinfel'd, "Quantum groups," in Proc. ICM (Berkeley, 1986), pp. 798-820, AMS, Providence, RI, USA, 1987.

[3] V. I. Arnold, Mathematical Methods of Classical Mechanics, vol. 60 of Graduate Texts in Mathematics, Springer, Berlin, Germany, 1978.

[4] P. Schaller and T. Strobl, "Poisson structure induced (topological) field theories," Modern Physics Letters A, vol. 9, no. 33, pp. 3129-3136, 1994.

[5] E. Frenkel and D. Ben-Zvi, Vertex Algebras and Algebraic Curves, vol. 88 of Math Surveys and Monographs, AMS, Providence, RI, USA, 2nd edition, 2004.

[6] M. Markl and E. Remm, "Algebras with one operation including Poisson and other Lie-admissible algebras," Journal of Algebra, vol. 299, no. 1, pp. 171-189, 2006.

[7] E. Remm, "Poisson superalgebras as nonassociative algebras," http://arxiv.org/abs/1205.2910v1.

[8] D. Yau, "Non-commutative Hom-Poisson algebra," 2012, http:// arxiv.org/abs/1010.3408.

[9] F. Ammar and A. Makhlouf, "Hom-lie superalgebras and HomLie admissible superalgebras," Journal of Algebra, vol. 324, no. 7, pp. 1513-1528, 2010.

[10] M. Goze and E. Remm, "Poisson algebras in terms of nonassociative algebras," Journal of Algebra, vol. 320, no. 1, pp. 294317, 2008.

[11] E. Remm, "Associative and Lie deformations of Poisson algebras," Communications in Mathematics, vol. 20, no. 2, pp. 117136, 2012. 


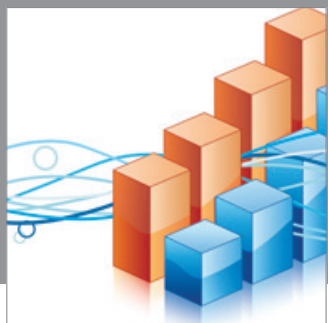

Advances in

Operations Research

mansans

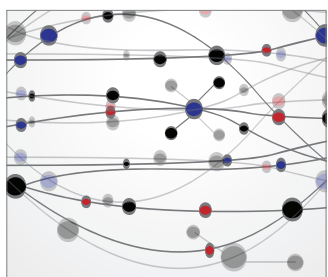

The Scientific World Journal
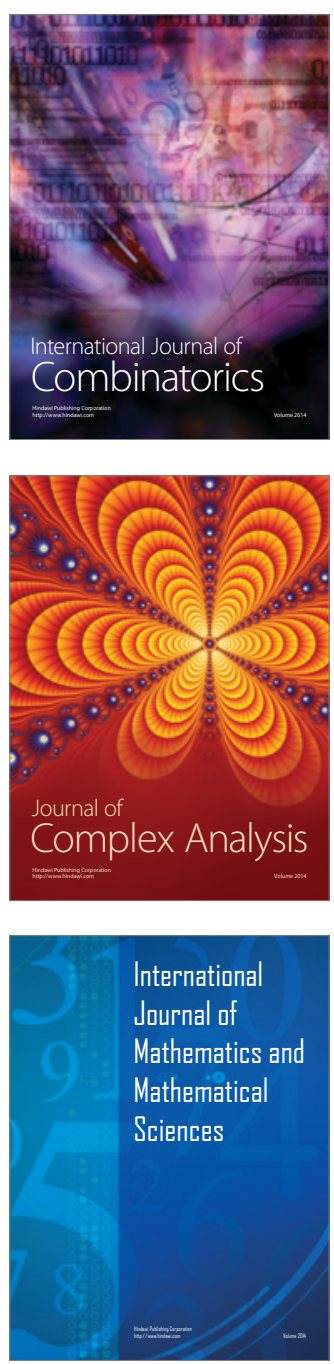
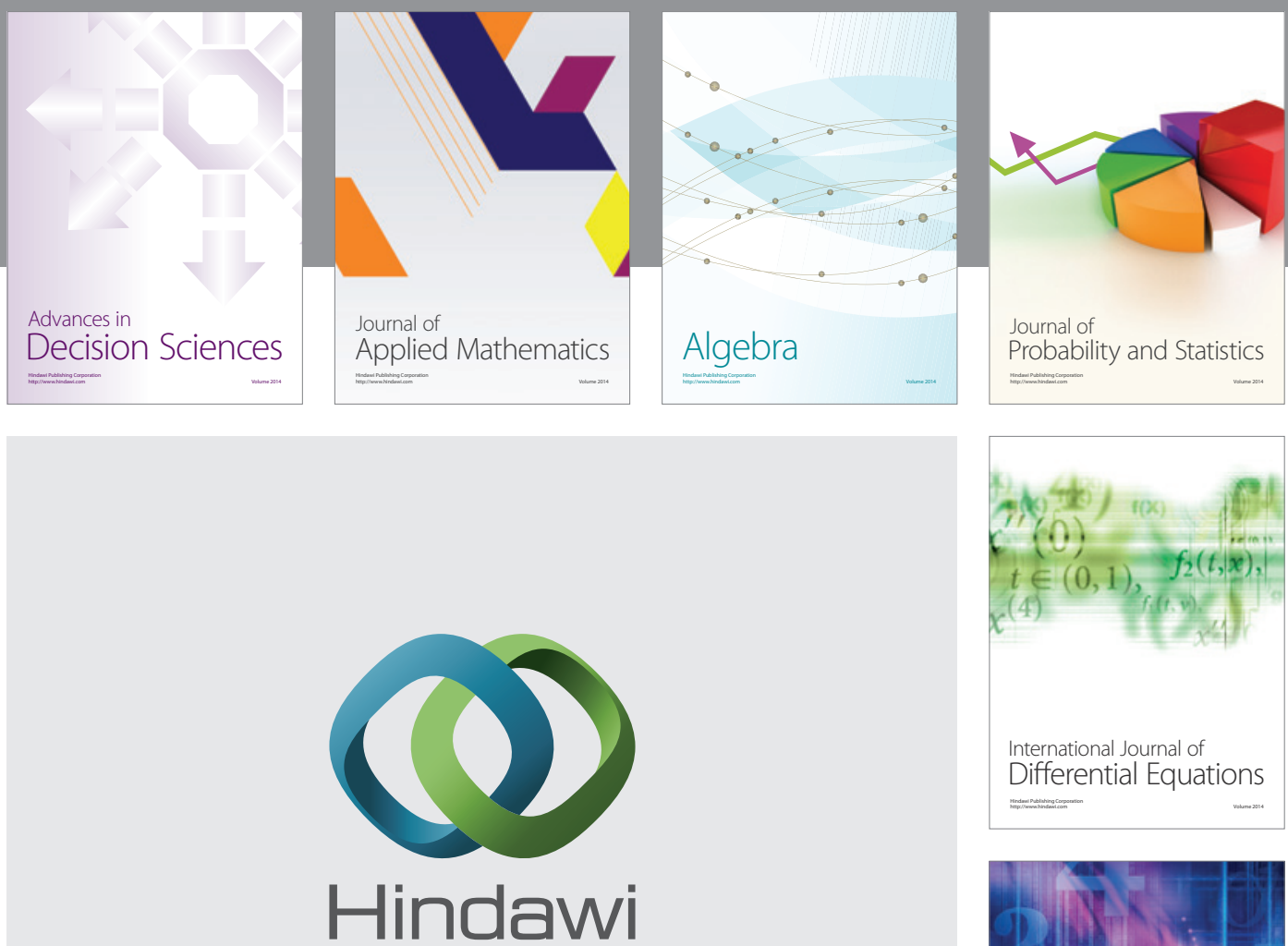

Submit your manuscripts at http://www.hindawi.com
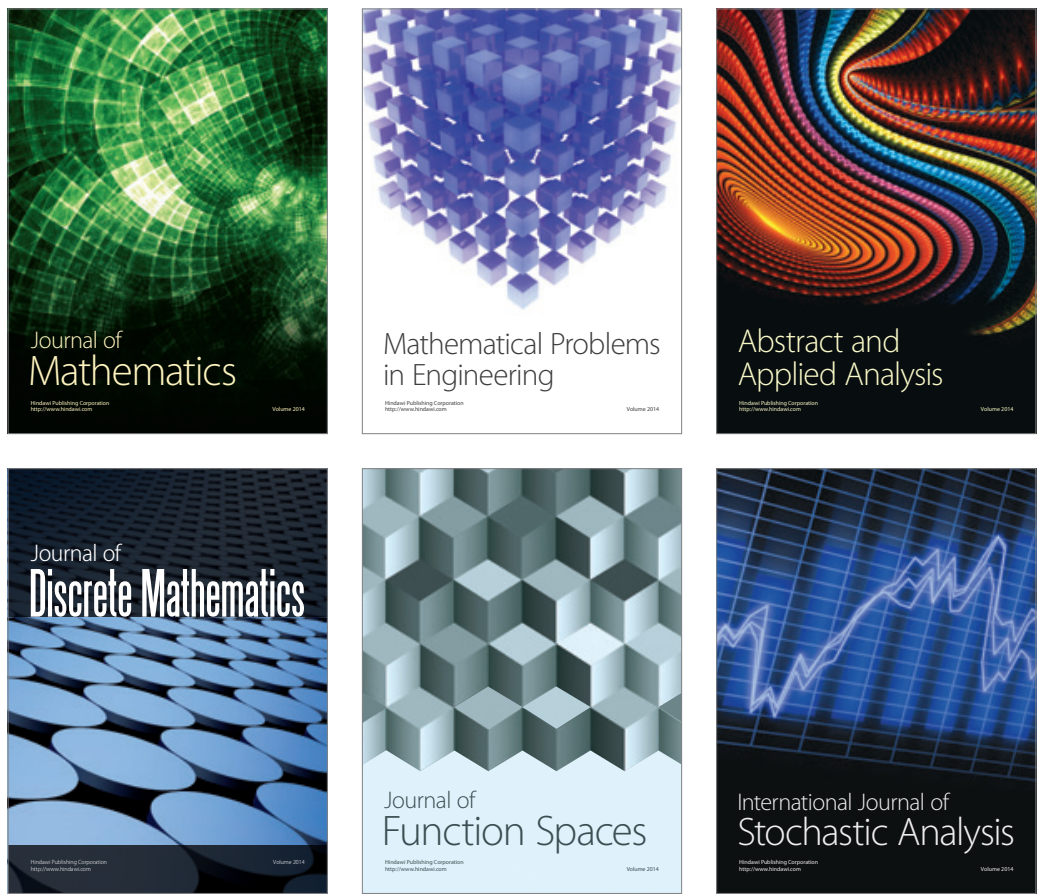

Journal of

Function Spaces

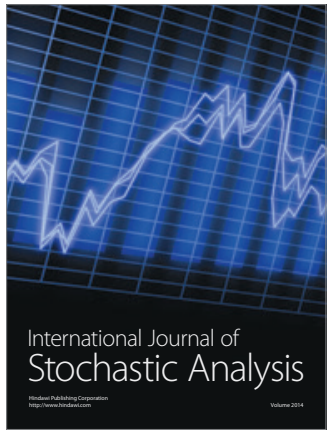

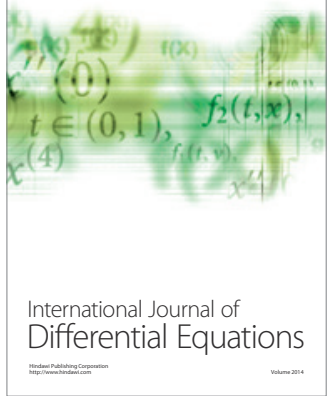
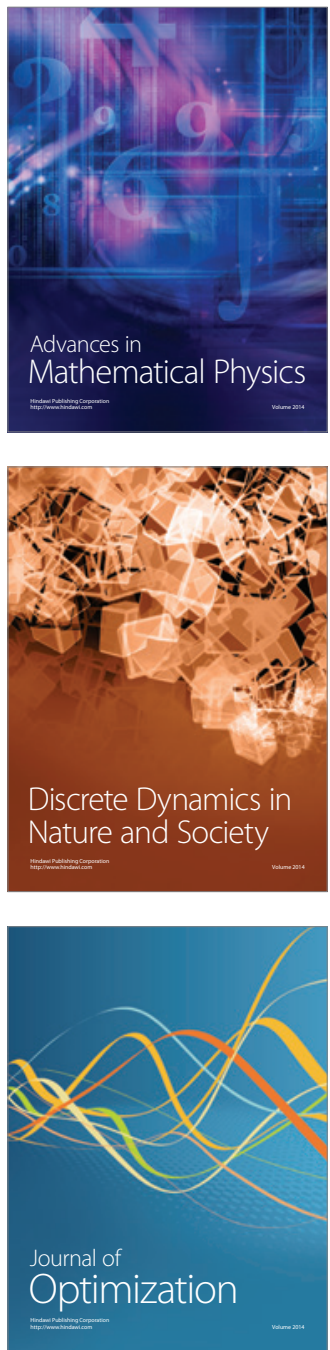